\title{
Bestimmung von Aminosäuren in Fruchtwasser- und Blutproben durch direkte quantitative Dünnschichtchromatographie mit elektronischer Auswertung
}

\author{
Von G. Kynast und J. W. Dudenhausen
}

Aus der Arbeitsgruppe Perinatale Mediqin (Leiter: Prof. Dr. E. Saling) der Freien Universität Berlin

(Eingegangen am 15. Juni/29. September 1972)

\begin{abstract}
Es wird eine direkte quantitative Bestimmungsmethode für Aminosäuren in Fruchtwasser- und Blutproben nach dünnschichtchromatographischer Trennung beschrieben. Die Auswertung wird mit einem dafür entwickelten Computer-Programm vorgenornmen. Die Messungen erfolgen mit einer Gerätekombination aus einem Spektralfotometer PMQ II-Zciss und einem Camag-Z-Scanner, die Auswertungen mit einem Tischrechner 9810 A Hewlett-Packard. Die relativen Standardabweichungen verschiedener Aminosäuren (Leu, Phe, Val, Ala, Thr) liegen im Mittel bei etwa 10\%, der Zeitbedarf bei Serienanalysen bei $4 \mathrm{~min} /$ Analyse, die Nachweisgrenze bei $0,05 \mu \mathrm{g} /$ Fleck (etwa $20 \mu \mathrm{mol} / \mathrm{l}$ ).
\end{abstract}

\section{Determination of amino acids in liquor amnii and blood samples by direct quantitative thin-layer chromatography and} data processing

A direct quantitative determination for amino acids in liquor amnii and blood samples is described. A specially developed computer programm is used for the evaluation. Measurements are carried out with apparatus consisting of a Spectral Photometer PMQ II-Zeiss combined with a Camag-Z-Scanner; a Hewlett-Packard 9810 A computer is used for the evaluation. The relative standard deviation of different amino acids (Leu, Phe, Val, Ala, Thr) amounts on average to approximately $10 \%$, the time required for series analyses to approximately $4 \mathrm{~min}$ per analysis and the identification limit to $0,05 \mu \mathrm{g} / \mathrm{spot}$ (about $20 \mu \mathrm{mol} / \mathrm{l}$ ).

Seit längerer Zeit werden bei uns Voruntersuchungen zur intrauterinen Ernährung von mangelversorgten Feten unternommen (1). Dabei müssen die Konzentrationen einer begrenzten Anzahl von Aminosäuren in vielen Fruchtwasserproben sowie mütterlichen und kindlichen Blutproben vor, während und nach der Behandlung hinreichend genau, möglichst schnell und mit geringem Laboraufwand bestimmt werden. Über die klinisch wichtigen Schlußfolgerungen aus den Ergebnissen wurde bereits berichtet (2). Auch die Resultate von Resorptions-Versuchen an frisch geborenen Nabelschnüren wurden veröffentlicht (3).

Sinn dieser Mitteilung ist es, genaue Angaben über die Einzelheiten der von uns entwickelten Meßmethode (Durchführung, Anwendungsbereich, zufällige und systematische Fehler, Nachweisgrenze, Zeitbedarf) zu machen. Die Grundlage bildet ein Verfahren, über das in anderem Zusammenhang bereits berichtet worden ist $(4,5)$.

\section{Methodik}

Prinzip

Das Analysenverfahren besteht aus vier Arbeitsgängen:

a) Vorbereitung der Analysen- und Eichproben

b) Behandlung und Entwicklung der Dünnschichtplatten

c) Messung der Analysen- und Eichflecke

d) Eingabe der Remissionswerte der Eichflecke in ein Computer-Programm zur Entwicklung einer Eichgeraden. Aus dieser wird durch Eingabe der Remissions- werte der Meßflecke der Gehalt der Analysenproben bestimmt.

\section{Reagenzien und Apparate}

Die als Eichstandards verwandten Aminosäuren L-Leucin, LPhenylalanin, L-Valin, L-Alanin und L-Threonin sind von der Fa. Merck, Darmstadt, bezogen worden. Damit werden gemischte Eichlösungen im Konzentrationsbereich von $0,0125 \mu \mathrm{g} / \mu \mathrm{l}$ bis $0,1 \mu \mathrm{g} / \mu \mathrm{l}$ (etwa $0,1 \mathrm{bis} 1 \mathrm{mmol} / \mathrm{l}$ ) je Aminosäure angesetzt. Als Laufmittel (A) dient eine Mischung aus n-Butanol/Aceton/Eisessig/Wasser (Volumina 3,5l+3,5l+1l+21). Zur Anfärbung wird ein weiteres Laufmittel (B) durch Zugabe von $15 \mathrm{ml} \mathrm{0,4} \mathrm{mol/1}$ Ninhydrinlösung (gelöst in A) auf $100 \mathrm{ml}$ Laufmittel A hergestellt.

Für die dünnschichtchromatographische Trennung werden $20 \times 20 \mathrm{~cm}$ große Dünnschicht-Fertigplatten der Fa. Merck, Darmstadt, mit $0,10 \mathrm{~mm}$ Cellulose-Beschichtung verwandt.

Eichlösungen und Analysenproben werden mit einer AglaMikrometerspritze aufgetragen, die zusammen mit einem in Höhe und Neigungswinkel verstellbaren Stativ von der Fa. Hormuth-Vetter, Wiesloch/Baden, bezogen wurde. Für die Auftragung und Behandlung der Platten wird ein Fön und gegebenenfalls eine IR-Lampe benutzt. Die Meßanordnung besteht aus einem Spektralfotometer PMQ Il der Fa. Zeiss, Oberkochen, und dem Dünnschicht-Z-Scanner der Fa. Camag, Muttenz/Schweiz. Für die Auswertung steht der Tischrechner $9810 \mathrm{~A}$ der Fa. HewlettPackard, mit Drucker, A]pha- und Statistik-Block zur Verfügung. Die für Auswertung und Fehlerberechnung erforderlichen Programme sind von uns entwickelt worden.

\section{Versuchsvorbereitung}

Auf den Dünnschichtplatten werden mit Bleistift je $2 \mathrm{~cm}$ breite Bahnen und in $3 \mathrm{~cm}$ Höhe die Startpunkte markiert. In $14 \mathrm{~cm}$ Höhe werden die Laufstreckenbegrenzungslinien mit einer Stahlnadel eingckratzt, so daß die Laufstrecke $11 \mathrm{~cm}$ beträgt. Von den 9 Bahnen werden 4 mit jc $3 \mu 1$ Eichlösung geeigneter Konzen- 
tration (z. B. 0,$02 ; 0,05 ; 0,10$ und $0,15 \mu \mathrm{g} / \mu \mathrm{l}$ Leu, Phe, Val, Ala, Thr) versehen. Auf die restlichen 5 Bahnen werden je nach Osmolarität und Gehalt 2 bis $5 \mu \mathrm{l}$ der Analysenproben aufgetragen. Die Mengen sollen so gewählt werden, daß Analysen- und Eichflecke gleiche Größe haben. Durch Vorversuche wurde sichergestellt, daß von den in meßbaren Mengen (Nachweisgrenze etwa $20 \mu \mathrm{mol} / 1)$ in Blut und Fruchtwasser vorkommenden Aminosäuren bei Verwendung des genannten Laufmittels keine Störungen hervorgerufen werden, die $R_{F}$-Werte sich also ausreichend unterscheiden. Bei Bestimmung anderer Aminosäuren müssen stets solche Vorversuche durchgeführt und das Laufmittel gegebenenfalls verändert werden. Zur Gewinnung schwebstofffreien Fruchtwassers, bzw. Serum- oder Plasma-Proben (für in vitro-Versuche bei vorher ungerinnbar gemachten Blutproben) werden die Fruchtwasser- oder Blutproben $10 \mathrm{~min}$ bei $3000 \mathrm{U} /$ min zentrifugiert. Vorversuche mit frischen Mustern zeigten keine signifikanten Unterschiede zwischen enteiweißten und nicht enteiweißten Proben. Im allgemeinen ist jedoch die Enteiweißung z. B. mit Sulfosalicylsäure $(50 \mathrm{~g} / \mathrm{l})$ ratsam.

Von unbehandelten Proben normaler Mütter und Feten werden bald nach ihrer Gewinnung unverdünnt $5 \mu \mathrm{l} /$ Fleck aufgetragen. EntsprechendeFruchtwasserproben erfordern wegen des geringeren Aminosäuregehaltes eine Auftragungsmenge von bis $\mathrm{zu} 20 \mu \mathrm{l} /$ Fleck, da die Nachweisgrenze bei den untersuchten Aminosäuren je nach Molekulargewicht bei $0,02 \mu \mathrm{g}$ ( $\mathrm{Ala}$ )/Fleck bis $0,1 \mu \mathrm{g}$ (Phe)/Fleck liegt, entsprechend etwa $20 \mu \mathrm{mol} /$ Fleck. Bei Proben, die nach intraamnialer Aminosäuregabe gewonnen wurden, müssen Bestimmungen aus Lösungen bis zu $5 \mathrm{mmol} / 1$, bei in vitro-Nabelschnurversuchen sogar bis zu $10 \mathrm{mmol} / 1$ durchgeführt werden, ivas eine starke Verdünnung der Meßproben erfordert.

Um eine optimale Genauigkeit zu erreichen, sollen die Eichlösungen so gewählt werden, daß die zu untersuchenden Proben im Eichbereich liegen. Bei Proben mit sehr geringem Aminosäuregehalt können größere Auftragungsmengen (bis $20 \mu \mathrm{l}$ ) gewählt werden. Es soll aber stets auf einheitliche Fleckengrößen geachtet werden. Dieses läßt sich durch Bestrahlung der Platte mit einer IR-Lampe während der Aufgabe realisieren. Nach vollständiger Trocknung der Flecke werden die Platten zuerst in Laufmittel A, nach Lufttrocknung dann in Laufmittel B entwickelt. Die Laufzeit beträgt jeweils etwa $2 \mathrm{~h}$, die $R_{\mathrm{F}}$-Werte liegen bei Leu/Phe/Val/Ala/Thr $=0,75 / 0,65 / 0,56 / 0,32 / 0,27$.

Vor Beginn der Auswertung läßt man die Platten zur Entwicklung der Ninhydrin-Färbung mindestens $2 \mathrm{~h}$ an der Luft trocknen. Durch Anblasen mit Warmluft (Fön) kann dieser Zeitraum erheblich verkürzt werden.

\section{Messung}

\section{Prinzip}

Für rein diffuse Reflexion gilt nach KubeLKa und Munk (6):

$$
F\left(R_{\infty}\right) \equiv \frac{\left(1-R_{\infty}\right)^{2}}{2 R_{\infty}}=\frac{k}{s}
$$

Dabei ist $\mathbf{R}_{\infty}$ das bei unendlicher Schichtdicke gemessene Reflexionsvermögen (Remissionswert), $\mathrm{k}$ der Absorptions- und s der Streukoeffizient. Für Dünnschichtchromatographie-Platten ist die Bedingung unendlicher Schichtdicke praktisch erfüllt. Zwischen den $\frac{k}{\mathrm{~s}}$-Werten und der Substanzmenge $\gamma$ in den Flecken besteht eine lineare Abhängigkeit, was sich auch in der Praxis in gewissen Grenzen bestätigt. So läßt sich aus den mitgelaufenen Eichlösungen eine Eichgerade $\gamma=\mathrm{F}\left(\frac{\mathrm{k}}{\mathrm{s}}\right)$ entwickeln. Dieser können die Gehalte der zu bestimmenden Flecke entnommen werden.
Umfangreiche Untersuchungen haben gezeigt (5), daß der Meßfehler geringer ist, wenn nicht der Remissionswert des gesamten Fleckenareals, sondern nur das mit flächenkonstantem Rechteckspalt $(3,5 \times 6 \mathrm{~mm})$ gemessene Remissionsminimum im Fleckenzentrum $R_{p}$ bestimmt wird. Neben $R_{p}$ wird der Remissionswert des Untergrundes $R_{u}$ gemessen. Diese Untergrundkorrektur ist gerade bei Messungen im sichtbaren Bereich nach Anfärbung wichtig, da der Untergrund an verschiedenen Stellen der Platte unterschiedlich gefärbt sein kann.

\section{Durchfübrung}

Mit der Gerätekombination PMQ II-Zeiss/Camag-Z̈-Scanner wird in der Anordnung Lampe-Monochromator-Probe-Empfänger, der Meßwellenlänge $523 \mathrm{~nm}$, dem Monochromatorspalt $0,5 \mathrm{~mm}$ und einer $3,5 \mathrm{~mm}$ hohen und $6 \mathrm{~mm}$ breiten Spaltblende gearbeitet. Die Empfindlichkeit des Verstärkerts wird so eingestellt, da $\dot{B}$ der Remissionsgrad für den Plattenuntergrund $R_{u}$ bei etwa $95 \%$ liegt. Zur Messung von $R_{p}$ im Fleckenzentrum wird der Rahmen mit den Dünnschichtchromatographie-Platten von Hand(!) so lange etwas horizontal und vertikal am Lichtspalt vorbeibewegt, bis das Anzeigegerät minimale Remission anzeigt. Dann wird die Platte quer zur Laufrichtung zum nächsten Fleck verschoben, wobei zwischen den Flecken $\mathrm{R}_{\mathrm{u}}$ abgelesen wird.

\section{Auswertung}

Aus den Meßwerten wird mit Hilfe eines Tischrechners der Gehalt der Analysenproben errechnet. Auf die Wiedergabe des Programms muß aus Platzgründen verzichtet werden, es kann auf Wunsch von den Autoren bezogen werden. Das Programm wird

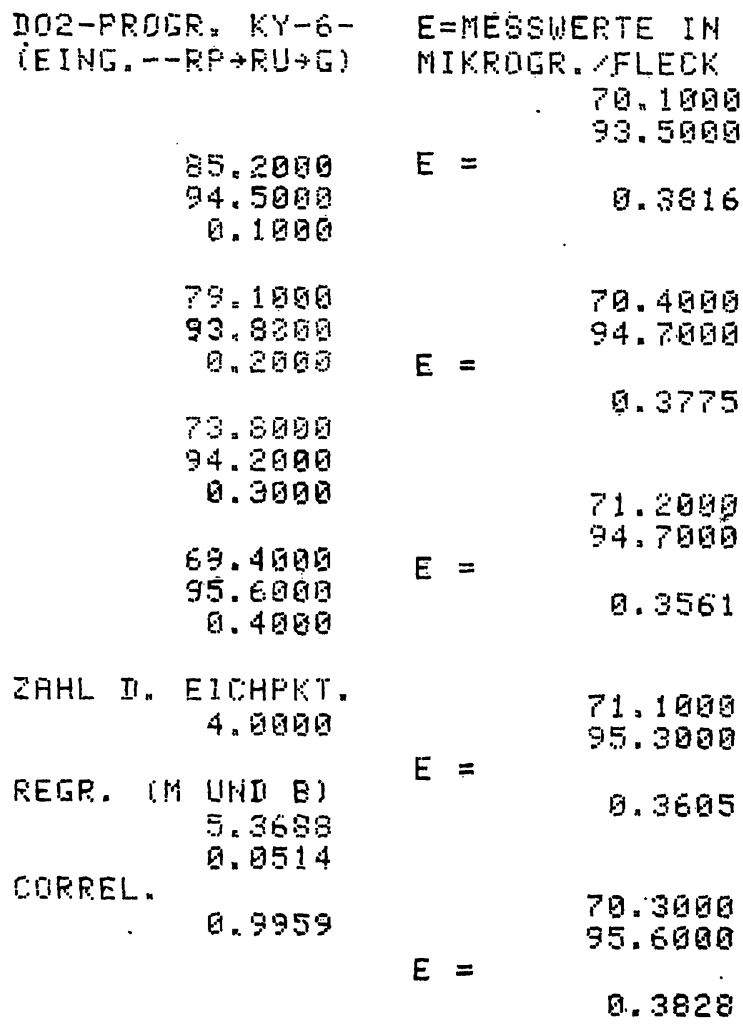

Abb. 1

Vom Tischrechner ausgedruckter Ergebriisstreifen für eine Dünnschichtchromatographie-Platte. Die linke Spalte zeigt im oberen Teil Remis(von oben nach unten): Remissionswert des Eichflecks (RP) Remis sionswert des Untergrunds (RU) und Einwaage pro Fleck (G). , Remisfolgen die Angaben über die Zahl der Eichpunkte, die errectinete Regressionsgerade (REGR.) mit Steigung M und Onte, die errechnet und der Korrelationskoeffizient (CORREL.). - Die rechte Spalte zeigt die eingegebenen Remissionswerte RP und $R U$ und die daraus errechneten Gehalte (E) für die 5 Meßflecke 
nach Drücken der Tasten RUN/END/PRGM in den Computer eingegeben und dann mit RUN/END/RECORD auf eine Magnetkarte übernommen. Nun kann es bei Bedarf mit RUN/END/ LOAD immer wieder in das Gerät eingelesen werden. Vor Eingabe der Meßwerte wird das Programm mit RUN/END/CONT aufgerufen. Zur Auswertung werden danach $R_{p}, R_{u}$ und $\gamma$ (in $\mu \mathrm{g}$ bzw. $\mu \mathrm{mol}$ ) jedes Eichfleckes in der angegebenen Reihenfolge in die Speicher $Z, Y$ und $X$ eingegeben (nach jeder Eingabe SHIFT UP drücken). Dann wird mit CONT die Eingabe der nächsten Dreiergruppe vorbereitet. Abbildung 1 zeigt im ersten Teil des ausgedruckten Streifens (linke Spalte) die Daten der nach Drücken von SET FLAG/CONT berechneten Eichgeraden und den zugehörigen Korrelationskoeffizienten. Ist dieser kleiner als 0,95 , so kann auf einen groben Fehler (Ausreißer) geschlossen werden. Die Eichgeradenberechnung sollte dann unter Weglassung dieses Meßwertes wiederholt werden. Danach werden $R_{p}$ und $R_{u}$ der Meßlecke in die Speicher $Y$ und $X$ (nach jeder Eingabe SHIFT UP drücken) gegeben. Mit CONT druckt der Computer dann die zugehörigen Gehalte in $\mu \mathrm{g}$ bzw. $\mu \mathrm{mol}$ aus (Abb. 1, rechte Spalte).

\section{Methodenprüfung}

Die in dieser Arbeit verwandte Nomenklatur zur Fehlererkennung wird entsprechend den vom Fachnormenausschuß Medizin im Deutschen Normenausschuß vorgeschlagenen Definitionen angewendet (7)

Tab. 1

Standardabweichungen für verschiedene Aminosäuren in einer Serumprobe

\begin{tabular}{|c|c|c|c|c|c|}
\hline & \multirow[t]{2}{*}{$\begin{array}{c}\text { Zahl der } \\
\text { Meßproben }\end{array}$} & \multicolumn{2}{|c|}{ Gefunden } & \multicolumn{2}{|c|}{$\begin{array}{l}\text { Standard- } \\
\text { abweichung }\end{array}$} \\
\hline & & [ $\mu \mathrm{g} /$ Fleck] & {$[\mu \mathrm{g} / \mu \mathrm{l}]$} & $\stackrel{\mathrm{s}_{\mathrm{a}}}{[\mu \mathrm{g} / \mu \mathrm{l}]}$ & $\begin{array}{c}s_{r} \\
{[\%]}\end{array}$ \\
\hline Leu & 35 & 0,3946 & 0,0789 & 0,0063 & 7,9 \\
\hline Phe & 39 & 0,3801 & 0,0760 & 0,0081 & 10,7 \\
\hline Val & 39 & 0,3981 & 0,0796 & 0,0074 & 9,3 \\
\hline Ala & 39 & 0,4345 & 0,0869 & 0,0121 & 13,9 \\
\hline Thr & 39 & 0,5448 & 0,1090 & 0,0204 & 18,7 \\
\hline
\end{tabular}

Tab. 2

Standardabweichungen für verschiedene Aminosäuren in einer Fruchtwasserprobe

\begin{tabular}{|c|c|c|c|c|c|}
\hline & \multirow[t]{2}{*}{$\begin{array}{c}\text { Zahl der } \\
\text { Meßproben }\end{array}$} & \multicolumn{2}{|c|}{$\begin{array}{c}\text { Gefunden } \\
\overline{\mathbf{x}}\end{array}$} & \multicolumn{2}{|c|}{$\begin{array}{c}\text { Standardab- } \\
\text { weichung }\end{array}$} \\
\hline & & [ $\mu \mathrm{g} /$ Fleck] & {$[\mu \mathrm{g} / \mu \mathrm{l}]$} & $\begin{array}{c}\mathrm{s}_{\mathrm{a}} \\
{[\mu \mathrm{g} / \mu \mathrm{l}]}\end{array}$ & $\begin{array}{c}s_{r} \\
{[\%]}\end{array}$ \\
\hline Leu & 35 & 0,0738 & 0,0185 & 0,0014 & 7,6 \\
\hline Phe & 35 & 0,0704 & 0,0176 & 0,0018 & 10,1 \\
\hline Val & 40 & 0,1129 & 0,0282 & 0,0019 & 6,8 \\
\hline Thr & 35 & 0,0845 & 0,0211 & 0,0027 & 12,8 \\
\hline
\end{tabular}

Tabelle 1 zeigt die für eine Serumprobe erhaltenen mittleren Aminosäuregehalte $\overline{\mathrm{x}}(\mu \mathrm{g} /$ Fleck und $\mu \mathrm{g} / \mu \mathrm{l})$ und die absoluten $\left(s_{a}\right)$ und relativen $\left(s_{r}\right)$ Standardabweichungen. $\mathrm{s}_{\mathrm{r}}$ liegt im allgemeinen bei etwa $10 \%$.

Tabelle 2 zeigt entsprechende Werte für eine Fruchtwasserprobe. Die erhaltenen Standardabweichungen liegen in der gleichen Größenordnung.

Tabelle 3 gibt die Ergebnisse einer Untersuchung wieder, die an einer künstlichen Testlösung mit einem Aminosäure-Gehalt von jeweils $0,050 \mu \mathrm{g} / \mu \mathrm{l}$ durchgeführt wird. Dieser Versuch dient der Berechnung des systematischen Fehlers (Abweichung des Meßwertes vom wahren Wert). Die Abschätzung des systematischen Fehlers ist notwendig, um die Richtigkeit der Ergebnisse sicherzustellen.

Das untersuchte Muster enthält von den Aminosäuren Leu, Phe, Val, Ala und Thr jeweils $0,050 \mu \mathrm{g} / \mu \mathrm{l}$. Auch hier liegen die Standardabweichungen bei etwa $10 \%$. Die Standardabweichungen der Aminosäure-Bestimmungen in physiologischen und künstlichen Lösungen liegen also in der gleichen Größenordnung. Das beweist, daß bei der Bestimmung im Plasma, Serum und Fruchtwasser keine zusätzlichen Fehler auftreten. Die Abweichung vom wahren Wert beträgt nur $2-4 \%$, womit die Richtigkeit der Ergebnisse bestätigt wird.

Der Arbeitsaufwand läßt sich entsprechend den bereits veröffentlichten Prinzipien (5) aufgliedern: Von einer Arbeitskraft können pro Arbeitstag (8 Stunden) bei der Bestimmung von z. B. 5 Aminosäuren 4 Dünnschichtplatten ausgewertet werden, das sind bei 5 Meßflecken 25 Analysen/Platte entsprechend 100 Analysen/Tag. Verteilt man den Zeitaufwand anteilig auf jede Analyse, so ergeben sich die in Tabelle 4 gezeigten Zeiten pro Platte. Pro Analyse sind 3 bis 4 Minuten erforderlich. Hinzu kommt die hier wegen der Verwendung polarer Laufmittel recht lange Laufzeit von etwa 4 Stunden/ Platte, in der jedoch keine Arbeitsleistung zu erbringen ist.

Tab. 4

Zeitbedarf für die quantitative Dünnschichtchromatographie

\begin{tabular}{lccccc}
\hline & $\begin{array}{c}\text { Vorbereitung } \\
\text { und } \\
\text { Auftragung }\end{array}$ & Messung & $\begin{array}{c}\text { Aus- } \\
\text { wertung }\end{array}$ & $\begin{array}{c}\text { Gesamt- } \\
\text { arbeits- } \\
\text { zeit }\end{array}$ & $\begin{array}{c}\text { Lauf- } \\
\text { zeit }\end{array}$ \\
\hline min/Platte & 30 & 50 & 10 & 90 & 240 \\
min/Analyse & 1,2 & 2,0 & 0,4 & 3,6 & \\
\hline
\end{tabular}

Tab. 3

Bestimmung des systematischen Fehlers in einer Testlösung

\begin{tabular}{|c|c|c|c|c|c|c|c|c|}
\hline & \multirow[t]{2}{*}{$\mathbf{n}$} & \multicolumn{2}{|c|}{ Wahrer Wert } & \multicolumn{2}{|c|}{ Gefundener Wert } & \multirow{2}{*}{$\begin{array}{c}\text { Abweichung } \\
\text { vom wahren Wert } \\
{[\%]}\end{array}$} & \multicolumn{2}{|c|}{ Standardabweichung } \\
\hline & & {$[\mu \mathrm{g} \mid \mu \mathrm{l}]$} & {$[\mathrm{mmol} / \mathrm{ll}]$} & {$[\mu \mathrm{g} / \mu \mathrm{l}]$} & [mmol/1] & & $\begin{array}{c}s_{\mathfrak{a}} \\
{[\mu \mathrm{g} / \mu \mathrm{l})}\end{array}$ & $\begin{array}{c}S_{\mathbf{r}} \\
{[\%]}\end{array}$ \\
\hline Leu & 14 & 0,050 & 3,81 & 0,0488 & 3,73 & 2,4 & 0,0058 & 11,8 \\
\hline Phe & 16 & 0,050 & 3,03 & 0,0479 & 2,90 & 4,2 & 0,0048 & 9,9 \\
\hline Val & 16 & 0,050 & 4,27 & 0,0484 & 4,13 & 3,2 & 0,0035 & 7,1 \\
\hline Ala & 7 & 0,050 & 5,61 & 0,0478 & 5,37 & 4,4 & 0,0035 & 7,4 \\
\hline Thr & $\cdot 14$ & 0,050 & 4,20 & 0,0509 & 4,27 & 1,8 & 0,0058 & 11,3 \\
\hline
\end{tabular}




\section{Diskussion}

Die Methode hat sich bei Untersuchung einer großen Anzahl von Blut- und Fruchtwasserproben bewährt. Die Nachweisgrenze liegt bei etwa $0,05 \mu \mathrm{g} /$ Fleck (etwa $20 \mu \mathrm{mol} / \mathrm{l})$. Der geringe Zeitaufwand von etwa $4 \mathrm{Minu}-$ ten pro Analyse macht es möglich, auch bei umfangreichem Versuchsmaterial jede Probe mehrfach zu analysieren (üblicherweise mindestens 3 Analysen/ Probe) und sich so gegen grobe Fehler (Ausreißer) abzusichern. Der Bedarf an Laborraum ist gering. Die verwendeten Geräte sind auch für andere Bestimmungsmethoden verwendbar. Die Ergebnisse fallen in übersichtlicher Form mit erläuterndem Text versehen auf Druckstreifen an und können als Dokument direkt abgelegt werden. Das Programm ist ohne wesentliche Änderungen auf die Bestimmung anderer Substanzen durch Remissionsmessung anwendbar. Ein weiterer Vorteil der Auswertung über ein Computer-Programm ist der, daß auch Messungen verwandt werden können, bei denen die Meßwerte weit außerhalb des Eichbereiches liegen. Das ist bei Proben mit unbekanntem Gehalt häufig der Fall. Die Ergebnisse sind dann weniger genau, erlauben aber eine Gehaltsabschätzung und Wiederholung der Messung im richtigen Bereich.

Zur Beurteilung der beschriebenen Methode soll ein Vergleich mit der allgemein üblichen säulenchromatographischen Bestimmung der Aminosäuren-Konzentrationen mit Aminosäure-Analysatoren durchgeführt werden. Dazu werden die Ergebnisse von verschiedenen Autoren herangezogen $(8,9,10,11)$.

Die Angaben über die Standardabweichungen für die Aminosäuren Leu, Phe, Ala, Val, Thr liegen bei $s_{\mathrm{r}}=3-27 \%$, sie werden bei den verschiedenen Autoren sehr unterschiedlich hoch angegeben. Die relativen Standardabweichungen bei der von uns benutzten Methode liegen im mittleren Bereich dieser Angaben. Aussagen über die sy'stematischen Fehler werden nicht gemacht. Aus der Tatsache, daß die bei den einzelnen Autoren angegebenen Mittelwerte teilweise weiter auseinander liegen als die relativen Standardabweichungen es zulassen, kann aber geschlossen werden, da $\beta$ die systematischen Fehler erheblich sind. Der Zeitbedarf wird mit 11 Stunden/Analyse angegeben (8). Umgerechnet auf jede der bestimmten 10-15 Aminosäuren ist das etwa eine Stunde/Aminosäuren-Bestimmung, wobei Vorbereitungs- und Auswertungszeit noch nicht eingeschlossen sind. Hier ist damit ein erheblicher Nachteil gegenüber der direkten quantitativen Dünnschichtchromatographie festzustellen. Gleiches läßt sich von den Kosten sagen, da der apparative Aufwand bedeutend größer ist.

Der Vergleich zeigt, daß die beschriebene Methode mit $10 \%$ einen im Durchschnitt höheren zufälligen Fehler als die Säulenchromatographie aufweist. Auch ist die Trennschärfe geringer, so daß sie sich weniger für die Bestimmung aller in Blut und Fruchtwasser in meßbaren Konzentrationen vorhandenen Aminosäuren eignet, als für die Bestimmung einer kleineren Gruppe. Das muß kein Nachteil sein, da häufig nur der Konzentrationsverlauf bestimmter Aminosäuren interessiert. Weiterhin kann die Trennschärfe durch Variation der Laufmittel, Verlängerung der Laufstrecke und zweimaliges Laufenlassen verbessert werden. Den Nachteilen der Dünnschichtchromatographie stehen als Vorteile gegenüber: wesentlich geringerer Zeitaufwand besonders bei Serienanalysen, beschleunigte objektive Auswertung durch Programmierung, vielseitige Anwendbarkeit, geringer systematischer Fehler und geringer Bedarf an Laborraum und Ausstattung.

\section{Danksagung}

Wir danken Herrn A. Eulenberger für seine Mitarbeit; dem Bundesministerium für Jugend, Familie und Gesundheit, BonnBad Godesberg, danken wir für die schnelle und großzügige Unterstützung.

\section{Literatur}

1. Saling, E., Salchow, P. \& Dudenhausen, J. W. (1972), in Perinatale Medizin II (Saling, E. \& Schulte, F. J., Hrsg.) S. 62, Thieme-Verlag, Stuttgart. - 2. Dudenhausen, J. W., KynaSt, G. \& Saling, E. (1972), in Perinatale Medizin III, (SALING E. \& Dudenhausen, J. W., Hrsg.) S. 230, Thieme-Verlag, Stuttgart. - 3. Dudenhausen, J. W., Kynast, G. \& Saling, E. Vortrag 3. Europäischer Kongreß für Perinatale Medizin, Lausanne, April 1972. - 4. KYNAST, G. (1970), Chromatographia 3 425-430. - 5. Krnast, G. (1971), Fresenius Z. Anal. Chem.
256, 20-24. - 6. Kortüм, G. (1962), Kolorimetrie, Photometrie und Spektralphotometrie, S. 357, Springer-Verlag, BerlinHeidelberg-New York. - 7. Fachnormenausschuß Medizin im Deutschen Normenausschuß, Qualitätskontrolle in der Laboratoriumsmedizin, DIN 58936, Blatt 1 und 2, September 1971. - 8. Liappis, N. (1972), diese Z. 10,132-135. - 9. Breuer, J., Kaese, G. \& Breuer, H. (1968), diese Z. 6, 298-303. - 10. GeroK, W. (1960), Klin. Wochenschr. 38, 1212-1215. - 11. OEPEN, H. \& Oepen, I. (1963), Klin. Wochenșchr. 41, 1048-1050. 